\title{
Iluminação artificial e orientação dos filhotes de Eretmochelys imbricata (Linnaeus, 1766), litoral sul de Pernambuco, Brasil
}

A poluição luminosa vem afetando diretamente o processo natural da orientação dos filhotes de tartarugas marinhas, que são atraídos pela luz e acabam seguindo em direção oposta ao oceano. Diante disso, o presente estudo objetivou avaliar se o processo natural de orientação de filhotes de Eretmochelys imbricata é influenciado pela presença de iluminação artificial e indicar pontos críticos quanto à iluminação no município do Ipojuca, Pernambuco, Brasil. A orientação dos filhotes foi analisada através de uma arena circular de $4 \mathrm{~m}$ de diâmetro, onde os filhotes eram colocados no centro e seus rastros eram traçados para registro de seus movimentos e, de acordo com a angulação de seus rastros na arena, foi possível classificá-los em 'orientados', 'desorientados' e com 'falta de orientação'. Também foi medida a intensidade luminosa no local. Foram evidenciadas diferenças significativas entre o processo de orientação e a presença/ausência de iluminação artificial para todos os casos testados. Para fatores abióticos avaliados, houve correlação entre a intensidade luminosa e o processo de orientação, mostrando que a iluminação artificial local vem afetando a vida dos filhotes. Em relação à lua, houve alguns registros de filhotes orientados no claro em noites com a presença lunar, mostrando uma importante influência da lua nesse processo, mas para a maioria dos casos, a luz da lua não foi o suficiente para restaura o processo natural de orientação dos filhotes. Além disso, foram categorizados 13 pontos como 'Muito crítico' e sete como 'Crítico' de acordo com a intensidade luminosa no local, evidenciando a necessidade de políticas ambientais locais e ações conservacionistas.

Palavras-chave: Conservação; Foto Poluição; Impacto Ambiental; Tartaruga de Pente.

\section{Artificial lighting and orientation in hatchlings of Eretmochelys imbricata (Linnaeus, 1766), south of Pernambuco, Brazil}

\begin{abstract}
Light pollution directly affects the natural orientation process of sea turtles' hatchlings that are attracted by artificial light and end up following to the opposite direction of the ocean. Therefore, the present study aimed to analyse whether the natural process of Eretmochelys imbricata hatchlings is influenced by the presence of artificial lighting and to indicate critical points of light pollution in municipality of Ipojuca, Pernambuco, Brazil. We analysed hatchlings' orientation through a circular arena, and we placed the animals in the middle, and analysed their trails according to the angles and then they were classified in 'oriented', 'disoriented' and 'miss-oriented'. Also, the luminous intensity was measured on site. We observed significant differences between the sea turtle orientation process and the presence/absence of artificial lighting for all tested cases. For the abiotic factors evaluated, there was a correlation between the light intensity and the orientation process, showing that the local artificial lighting is affecting the hatchlings lives. For the moon light presence, there were records of hatchlings that have been oriented by the lunar light in the presence of artificial lighting, showing a significant influence of the moon in this process. However, the lunar light was not enough to restore the hatchlings natural process of orientation in most of the cases. Additionally, 13 illuminated points were categorized as 'Very critical' and seven as 'Critical' in relation to the luminous intensity, emphasizing the needs for local environmental policies and conservation actions in the region.
\end{abstract}

Keywords: Conservation; Environmental Impact; Hawksbill Turtles; Light Pollution.

Topic: Conservação da Biodiversidade

Reviewed anonymously in the process of blind peer
Received: 02/05/2020

Approved: 01/06/2020
Ana Elisabeth Cordeiro Sayegh (ID

Universidade Federal Rural de Pernambuco, Brasil

http://lattes.cnpq.br/3790882905357413

http://orcid.org/0000-0003-2538-383X

sayegh.ana@gmail.com

Thyara Noely Simões (iD)

Universidade Estadual de Santa Cruz, Brasil

http://lattes.cnpq.br/47182887768887692

http://orcid.org/0000-0001-8633-2169

thyara.noely@gmail.com

Ednilza Maranhão dos Santos (iD

Universidade Federal Rural de Pernambuco, Brasi

http://lattes.cnpq.br/5812920432455297

http://orcid.org/0000-0001-9214-1131

ednilzamaranhao@gmail.com
Arley Cândido da Silva

Universidade Norte do Paraná, Brasil

http://lattes.cnpq.br/6695684507238704

ecoassociados.projeto@gmail.com
Referencing this:

SAYEGH, A. E. C.; SIMÕES, T. N.; SANTOS, E. M.; SILVA, A. C.. Iluminação artificial e orientação dos filhotes de Eretmochelys imbricata (Linnaeus, 1766), litoral sul de Pernambuco, Brasil. Revista Ibero Americana de Ciências Ambientais, v.11, n.4, p.89-101, 2020. DOI: http://doi.org/10.6008/CBPC2179-6858.2020.004.0008

DOI: $10.6008 / C B P C 2179-6858.2020 .004 .0008$ 


\section{INTRODUÇÃO}

As principais causas de declínio das populações de tartarugas marinhas estão relacionadas à perda de habitat, a pesca industrial e artesanal, o abate das fêmeas, a ingestão de resíduos inorgânicos, como o plástico, a predação dos ovos, industrialização das zonas costeiras e os efeitos da urbanização, principalmente no que se refere à iluminação artificial (MORTIMER et al., 2008). Em um caso descrito por McFarlane (1963) na Flórida, 95\% dos filhotes de Caretta caretta de um ninho morreram de exaustão ou atropelados devido a desorientação causada pela presença de iluminação artificial no ambiente. Isso ocorre porque os filhotes ao emergirem dos ninhos têm como orientação o ponto mais brilhante do horizonte, que atualmente, vem sendo a iluminação deixada pelas casas, grandes hotéis, condomínios, bares e outras construções a beira mar (MROSOVSKY et al., 1985). Como consequência, os filhotes se tornam vulneráveis a desidratação e predadores, além de poderem ir a óbito por exaustão (SALMOM, 2003; BOURGEOIS et al., 2009; ZHELEVA, 2012). A desorientação de filhotes devido à poluição luminosa nas praias urbanizadas também já foi reportada para Chelonia mydas (MROSOVSKY et al., 1967; PENDOLEY et al., 2016), C. Caretta (SALMON et al., 1995; PRICE et al., 2018), Lepidochelys olivacea (KARNAD et al., 2009; CRUZ et al., 2018), Dermochelys coriacea (BOURGEOIS et al., 2009; RIVAS et al., 2015) e Natator depressus, espécie endêmica da Austrália (PENDOLEY et al., 2015).

O litoral sul de Pernambuco representa atualmente, um dos principais polos turísticos da região nordeste do Brasil, aumentando a especulação imobiliária e promovendo maior urbanização na sua orla com a predominância de condomínios e hotéis (LIMA, 2006). Concomitantemente é uma importante área de nidificação de Eretmochelys imbricata além de haver registros de desovas de outras três espécies (C. caretta, L. olivacea e C. mydas), todas possuindo alguma categoria de ameaça à extinção (IUCN, 2019). A espécie com maior número de desovas nesse litoral é E. imbricata (MOURA et al., 2012), que pode estar sofrendo ameaça quanto à orientação dos filhotes.

A espécie E. imbricata possui a categoria mais elevada dentro da lista vermelha para espécies ameaçadas em extinção feita pela IUCN (IUCN, 2019). Na lista vermelha brasileira, feita pelo Ministério do Meio Ambiente, a espécie também se encontra na categoria mais elevada, criticamente em risco de extinção (MMA, 2018). Essa espécie nidifica em 76 países, incluindo a costa brasileira (MORTIMER et al., 2008). De acordo com Marcovaldi et al. (2011), no Nordeste brasileiro, E. imbricata apresenta como área prioritária de nidificação o litoral norte do estado da Bahia e Sergipe e litoral sul do Rio Grande do Norte. Além disso, o estado de Pernambuco, principalmente o litoral Sul, constitui uma área significativa de nidificação dessa espécie (MOURA et al., 2012; SIMÕES et al., 2014), todavia há apenas um registro que relata os impactos sobre os filhotes quanto à presença de iluminação na área (SIMÕES et al., 2017). Os trabalhos de conservação desses animais no estado se concentram nas praias do município do Ipojuca, onde um total de $12 \mathrm{~km}$ de faixa litorânea é monitorado pela Organização Não Governamental Ecoassociados (SIMÕES et al., 2014).

As políticas ambientais no que se refere à poluição luminosa em áreas litorâneas são de extrema importância para a sobrevivência e conservação de animais noturnos, principalmente as tartarugas marinhas, 
que são vulneráveis as ações antrópicas. Atualmente, o TAMAR dispõe de uma Cartilha de Foto poluição, onde é explicada a ameaça que a iluminação artificial pode causar as tartarugas marinhas e são mostrados os tipos adequados e não adequados de iluminação que se deve ter na região da Bahia (SALIÉS, 2014). Além disso, existe no Brasil a Portaria no 11, de 30 de janeiro de 1995 (BRASIL, 1995), que proíbe qualquer fonte de iluminação com intensidade luminosa superior a zero lux em áreas de desovas de tartarugas marinhas. Porém apenas algumas praias estão submetidas a essa legislação, em especial as áreas protegidas, como as Unidades de Conservação, a exemplo do arquipélago de Fernando de Noronha em Pernambuco.

Diante do exposto, o presente estudo objetivou avaliar se o processo natural de orientação de filhotes de E. imbricata no litoral do município do Ipojuca em Pernambuco é influenciado pela presença de iluminação artificial. Simultaneamente, observar se parâmetros abióticos como temperatura $\left({ }^{\circ} \mathrm{C}\right)$, umidade relativa do ar (UR\%), velocidade do vento $(\mathrm{Km} / \mathrm{h})$ e fase lunar também podem exercer alguma influência nesse processo. Além disso, mapear os pontos de poluição luminosa, destacando aqueles de maior gravidade com base na legislação brasileira existente e sugerir medidas de conservação e de gestão pública.

\section{MATERIAIS E MÉTODOS}

\section{Área de estudo}

A área de estudo se localiza no litoral Sul do estado de Pernambuco, Brasil, mais precisamente no município do Ipojuca, cerca de $50 \mathrm{~km}$ de distância da capital Recife. Dos $32 \mathrm{~km}$ de faixa de praia do município, o estudo se concentrou em $12 \mathrm{~km}$ que são monitorados pela ONG Ecoassociados que trabalha com conservação e reabilitação das tartarugas marinhas na região. Os $12 \mathrm{~km}$ de praias monitoradas correspondem as praias de Muro Alto, Cupe, Merepe, Porto de Galinhas e Maracaípe (SIMÕES et al., 2014; SILVA et al., 2019) (Figura 1).

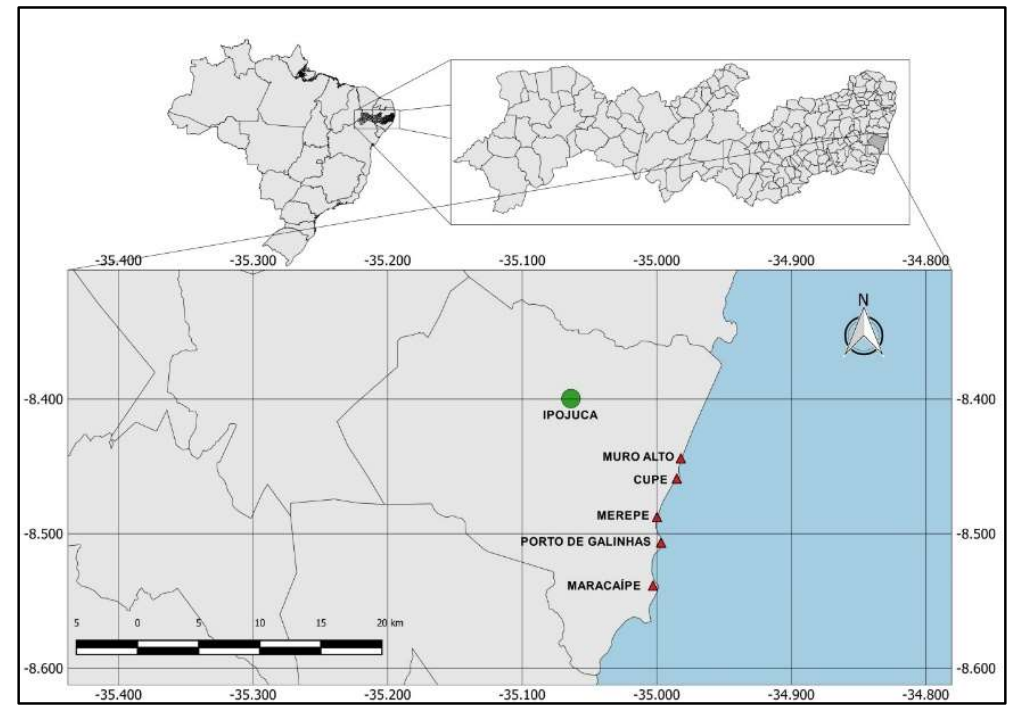

Figura 1: Mapa de localização da área de estudo em Ipojuca/PE, evidenciando as praias onde há nidificação de tartarugas marinhas que são monitoradas pela ONG Ecoassociados. Fonte: Silva et al. (2019).

As praias do município do Ipojuca são áreas de preferência para a desova de tartarugas marinhas em Pernambuco (GUIMARÃES et al., 2010). De acordo com Moura et al. (2012) as praias de Muro Alto e Cupe apresentam um pequeno banco recifal. A praia de Merepe, no entanto, não apresenta barreiras de acesso à 
praia, o que faz com que seja a praia com o maior registro de desovas. A praia de Porto de Galinhas apresenta o menor registro de desova devido à presença de um grande recife de coral. Já a praia de Maracaípe fica próxima a uma região de estuário, com a presença de recifes de arenito margeando a costa de forma irregular. Todas essas praias apresentam características ambientais como temperatura, umidade, tipo de areia e topografia favoráveis à escolha do ambiente para a desova de tartarugas marinhas. Porém, todas as praias sofrem influência direta de condomínios, hotéis e pousadas (MOURA et al., 2012).

\section{Procedimentos metodológicos}

Os experimentos foram realizados entre fevereiro e maio de 2016. Um total de 20 ninhos foram escolhidos aleatoriamente para fazer parte da amostra experimental, sendo 10 deles analisados na presença de iluminação artificial e 10 na ausência dessa iluminação. Foi desenhada na areia da praia uma arena circular de $4 \mathrm{~m}$ de diâmetro em locais onde houve desovas durante a temporada reprodutiva de 2015/2016. No centro da arena foi criada uma depressão de 2 a $3 \mathrm{~cm}$ para simular as condições naturais de emersão dos filhotes. O centro da arena, em direção ao oceano, foi considerado como ângulo 0 on, seguindo as orientações proposta por Salmon et al. (1995). Foi utilizado um total de 66 filhotes por ninho para cada experimento. Em cada experimento, os testes foram repetidos três vezes com 7 filhotes e três vezes com 15 filhotes. Os filhotes foram colocados no centro da arena onde se observou a influência da presença ou ausência da iluminação artificial na sua orientação. Após a ultrapassagem da extremidade da arena, foi medida a angulação dos rastros dos filhotes, categorizando-os de acordo com os processos de orientação. Concomitantemente foi fotografado e o traçado dos animais foi ilustrado em um modelo esquemático a fim de registrar os movimentos circulares ou mudanças de direção que surgiram, identificando o grau de desorientação por determinada intensidade luminosa.

De acordo com a metodologia proposta por Salmon et al. (1995), a direção dos filhotes em relação ao oceano é considerada rompida quando eles têm um desvio de direção do mar maior que 30 tanto para direita como para esquerda, contando a partir do ângulo 0‥ Portanto, a direção dos filhotes foi dividida, com base no processo de orientação, em três categorias: orientados, desorientados e os com falta de orientação. Foram categorizados como 'orientados' os filhotes que tiveram desvio angular menor que 30, andando diretamente em direção ao oceano. Para a categoria 'desorientados', enquadraram-se os filhotes que andaram em círculos dentro da arena e que encontraram ou não o caminho para o oceano. Já a 'falta de orientação' foi assumida quando os filhotes andaram diretamente para uma direção com desvio angular maior que 30 ․

A intensidade luminosa dos pontos foi medida com o auxílio de um luxímetro, LM8000 Termo-HigroAnemometro-Luximetro digital, em todas as direções, norte, sul, leste, oeste e no centro da arena onde foi criada a depressão para simular as condições de saída do ninho. Para cada experimento foi registrado parâmetros como temperatura $\left({ }^{\circ} \mathrm{C}\right)$, umidade relativa do $\operatorname{ar}(\mathrm{UR} \%)$ e velocidade do vento $(\mathrm{Km} / \mathrm{h})$ através do luxímetro, além da fase lunar. Paralelamente os pontos iluminados foram mapeados, sendo georeferenciados, analisados e caracterizados quanto à sua intensidade luminosa. Esses pontos receberam 
a classificação de 'Muito crítico' e 'Crítico'. Os pontos 'Muito crítico' obtiveram intensidade luminosa superior a 0 lux, já os 'Crítico' obtiveram sua intensidade luminosa igual a 0 lux, porém há registros anteriores feitos pela ONG Ecoassociados de casos de filhotes andando em direção oposta ao mar, sendo encontrados nos jardins das casas, por exemplo. Além disso, foi aferida a distância dos ninhos para cada ponto luminoso com auxílio do Google Earth.

\section{Análise de dados}

Utilizou-se para análise dos dados a estatística descritiva, como frequência, média e desvio padrão. Em seguida, testou-se a normalidade aplicando o teste Kolmogorov-smirnov e Shapiro Wilk's, posteriormente, utilizou-se a correlação de Spearman $\left(r_{s}\right)$ Rank Order para correlacionar com fatores abióticos como temperatura, umidade relativa do ar, a velocidade do vento, a fase da lua e a intensidade média da iluminação artificial no local com o processo de orientação dos filhotes. Para testar diferenças entre o processo de orientação dos filhotes de ninhos iluminados e não iluminados (claro e escuro, respectivamente) e entre os experimentos com 7 e 15 filhotes, foi utilizado o test Mann-Whitney (U), ambos foram verificados através do programa Statistica 8.0 (StatSoft, 2013). Para todos os testes foram considerados valores estatisticamente significantes $\operatorname{com} p=\leq 0,05$.

\section{RESULTADOS}

No total, para os 10 experimentos no escuro e 10 no claro, foram utilizados 1.320 filhotes, onde $48,48 \%$ foram considerados como orientados (38,78\% para escuro e 9,70\% para claro), $11,52 \%$ como desorientados (1,97\% para escuro e 9,55\% para claro) e 40\% exibiram comportamento de falta de orientação (9,24\% para escuro e 30,76\% para claro). Houve diferenças significativas entre o processo de orientação e a presença ou ausência de iluminação artificial para todos os casos testados (Figura 2), com destaque para os orientados e falta de orientação quanto aos experimentos envolvendo 15 filhotes (Figura 3).

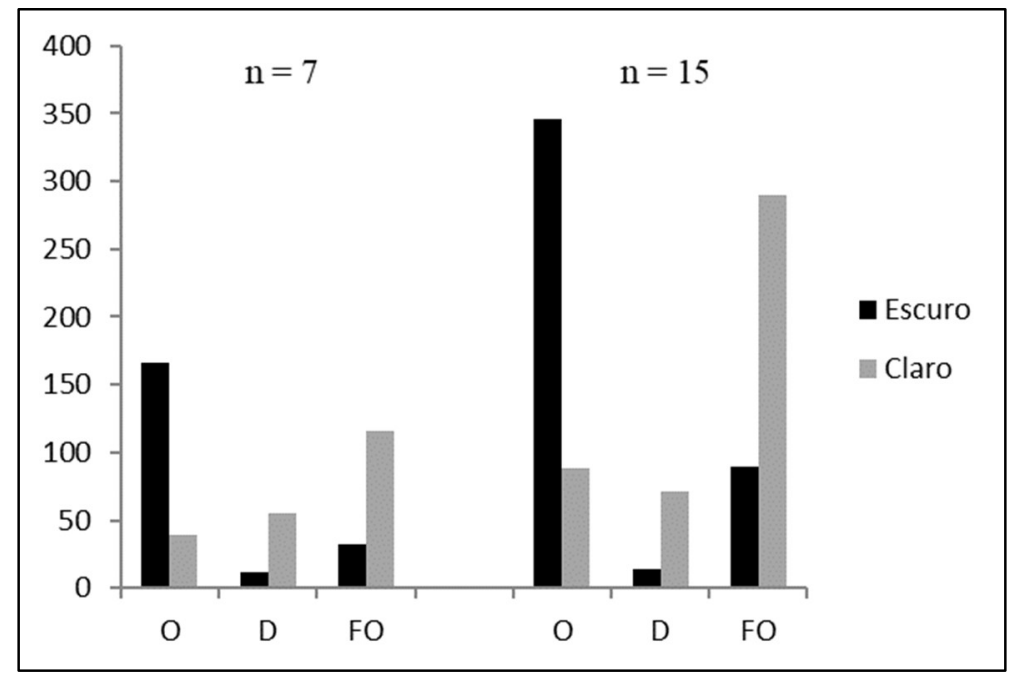

Figura 3: Quantidade de filhotes de E. imbricata em cada categoria de orientação ( $\mathrm{O}=$ Orientados; $\mathrm{D}=$ Desorientados; FO = Falta de orientação) para os experimentos realizados no escuro e no claro, com n=7 e $n=15$ filhotes em Ipojuca/PE. 


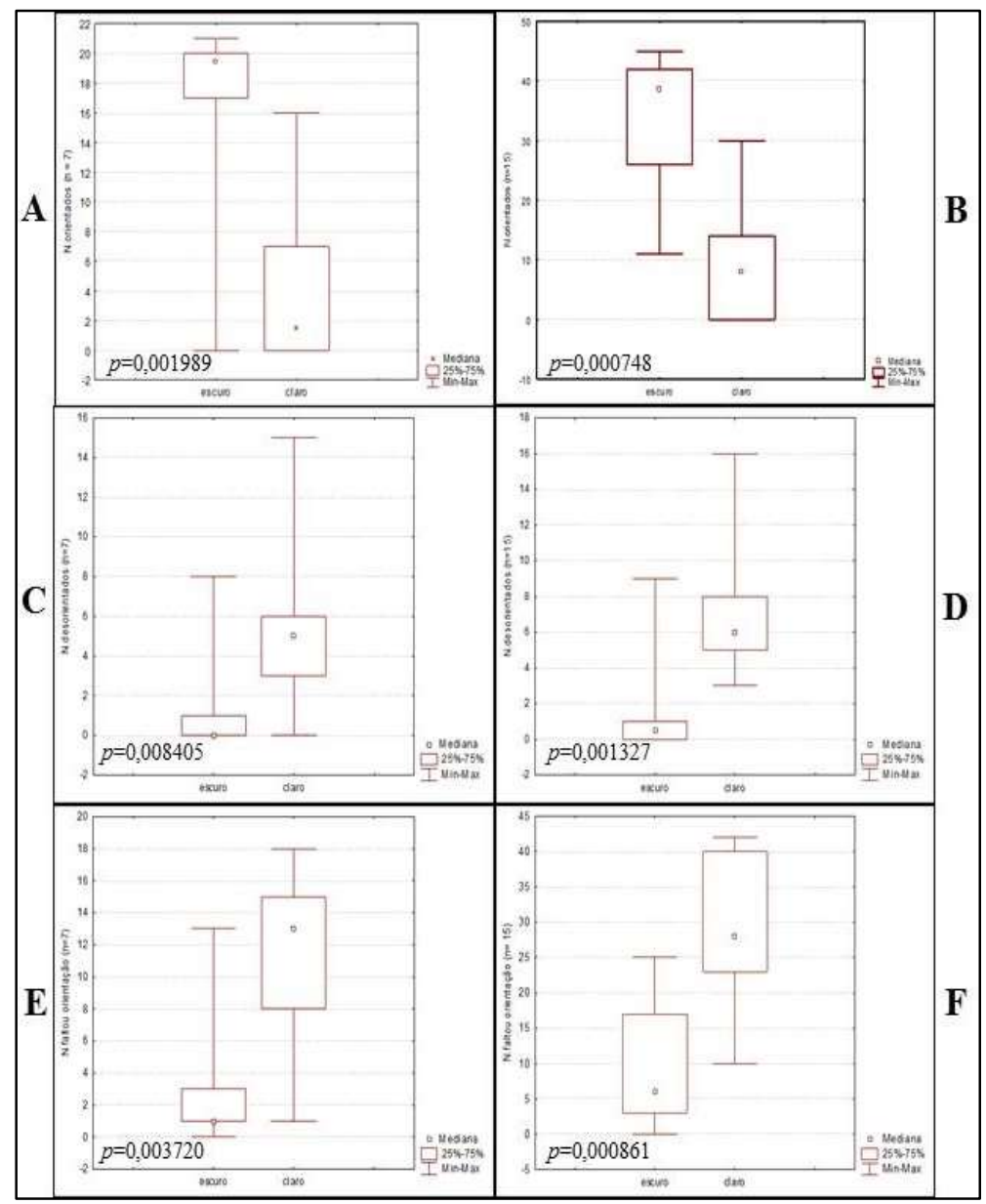

Figura 2: Diferenças entre os processos de orientação para experimento com $n=7$ e $n=15$ e entre escuro e claro em Ipojuca/PE. A - Diferença entre orientados n=7; B - Diferença entre orientados n=15; C - Diferença entre desorientados $n=7 ; D$ - Diferença entre desorientados $n=15$; $E$ - Diferença entre falta de orientação $n=7$; $F$ - Diferença entre falta de orientação $n=15$.

Não houve nenhuma correlação estabelecida entre alguns dos parâmetros abióticos analisados e as categorias de orientação. Porém, para intensidade luminosa houve correlação para todas as categorias, tendo uma relação inversa para orientados. Para a temperatura a correlação foi significativa, exceto para falta de orientação ( $n=7)$. Quanto às fases da lua foi obtida correlação direta para orientados ( $n=7$ e $n=15)$ e correlação indireta para desorientados $(n=15)$ e falta de orientação quando o experimento foi realizado com $\mathrm{n}=7$ filhotes (Tabela 1 ).

Tabela 1: Parâmetros abióticos e processos de orientação para os experimentos realizados com $n=7$ e $n=15$ filhotes de E. imbricata em Ipojuca/PE.

\begin{tabular}{|l|l|l|l|l|l|l|}
\hline \multirow{2}{*}{ Parâmetros } & Orientação & Desorientação & \multicolumn{2}{l|}{ Falta de orientação } \\
\cline { 2 - 7 } & $\mathbf{N = 7}$ & $\mathbf{N}=\mathbf{1 5}$ & $\mathbf{N}=\mathbf{7}$ & $\mathbf{N}=\mathbf{1 5}$ & $\mathbf{N}=\mathbf{7}$ & $\mathbf{N}=\mathbf{1 5}$ \\
\hline Lux & $-0,61788$ & $-0,68798$ & 0,47477 & 0,51174 & 0,65907 & 0,60739 \\
\hline Temperatura & $-0,64306$ & $-0,62268$ & 0,61538 & 0,56371 & - & 0,64248 \\
\hline Lua & 0,50912 & 0,47663 & - & $-0,58866$ & $-0,49173$ & - \\
\hline
\end{tabular}

Ao comparar o processo de orientação entre as fases da lua, para os experimentos claro e escuro, ficou evidente que um maior número de eventos foi registrado quando a lua estava crescente tanto para claro quanto para escuro, no entanto, no claro para falta de orientação e no escuro para orientados (Figura $4)$. 


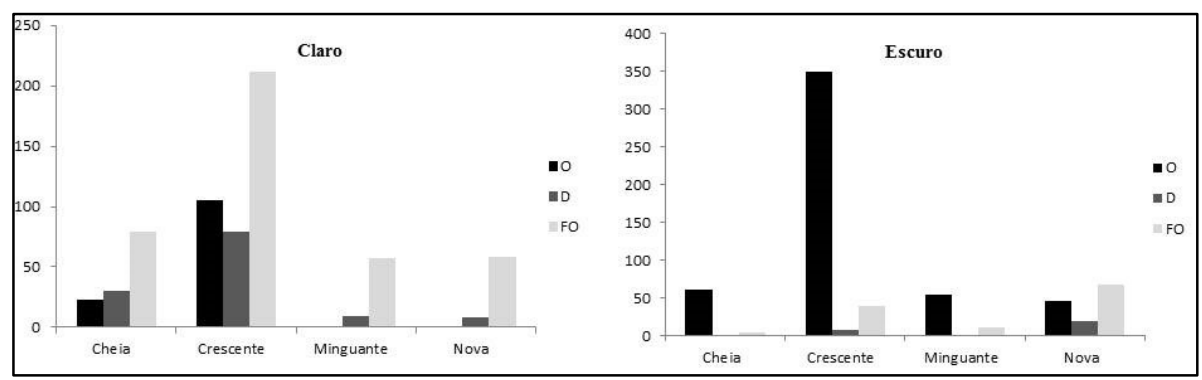

Figura 4: Distribuição das categorias de orientação para cada fase da lua nos experimentos realizados em Ipojuca/PE. $\mathrm{O}=$ Orientados; $\mathrm{D}=$ Desorientados e FO = Falta de orientação.

Um total de 20 pontos foram registrados e georreferenciados (Figura 5). A iluminação foi referente à 11 hotéis, 6 condomínios de casas, 2 postes de iluminação pública e uma residência particular. Dentre esses pontos, 7 foram considerados como 'Crítico' e 13 como 'Muito crítico', com destaque para Merepe com 6 pontos 'Muito crítico' e 5 'Crítico', seguido por Muro Alto com 4 pontos 'Muito crítico', Cupe com 1 ponto 'muito crítico' e 2 'Crítico' e Maracaípe com 2 'Muito crítico'. A intensidade luminosa entre os pontos variou entre 0 e 47 lux e a distância do ponto de iluminação ao ninho variou entre $0 \mathrm{~m}$, quando o ninho era localizado logo abaixo do ponto de iluminação, e $50 \mathrm{~m}$. A praia de Porto de Galinhas não entrou na amostra devido à ausência de desovas nessa praia durante a temporada reprodutiva de 2015/2016.

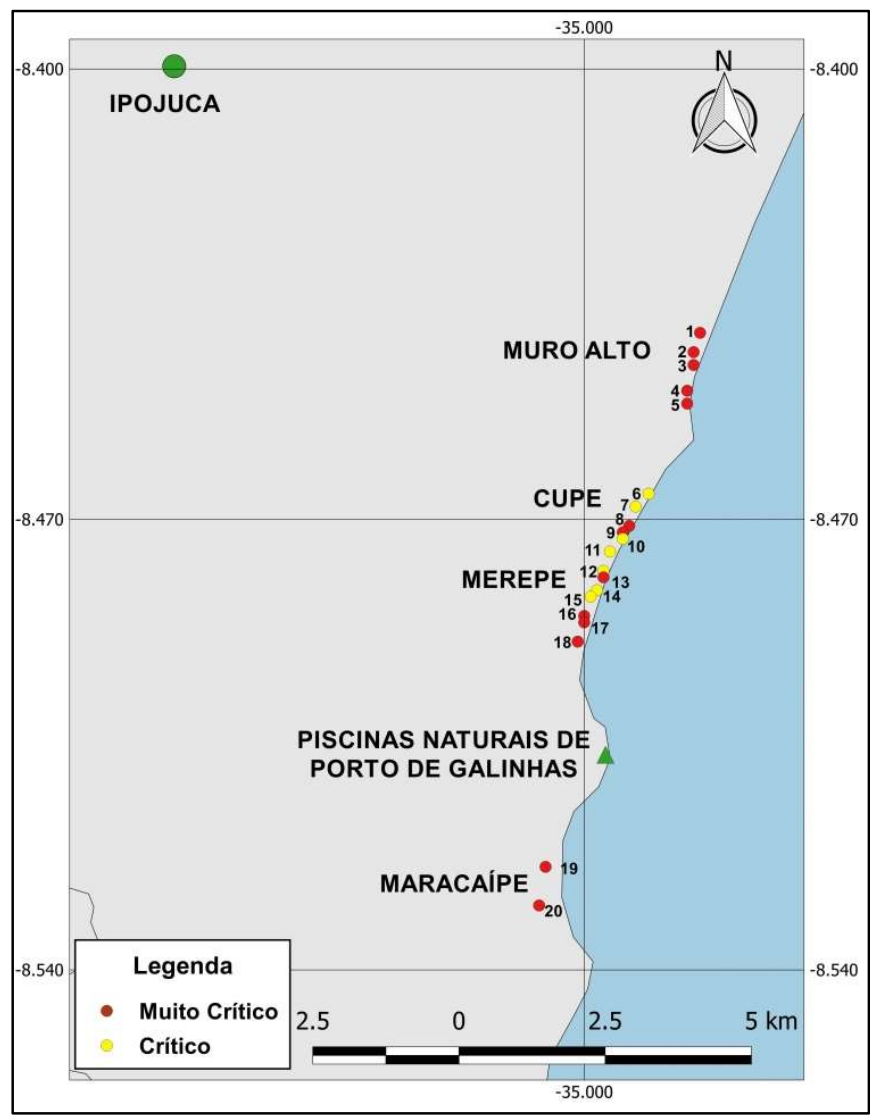

Figura 5: Distribuição dos 20 pontos iluminados ao longo das quatro praias estudadas em Ipojuca/PE.

Tabela 2: Dados da intensidade luminosa, distância entre a fonte de luz e a localização do ninho e categoria de avaliação de cada ponto na temporada de 2015/2016 em Ipojuca/PE.

\begin{tabular}{|l|l|l|l|}
\hline Ponto & Intensidade & Distância & Classificação \\
\hline 1 & 10 lux & $10 \mathrm{~m}$ & Muito crítico \\
\hline 2 & 2 a 47 lux & 0 a $5,5 \mathrm{~m}$ & Muito crítico \\
\hline 3 & 0 a 7 lux & 0 a $50 \mathrm{~m}$ & Muito crítico \\
\hline 4 & 2 a 17 lux & 0 a $7 \mathrm{~m}$ & Muito crítico \\
\hline
\end{tabular}




\begin{tabular}{|c|c|c|c|}
\hline 5 & $13 \operatorname{lu} x$ & $12 \mathrm{~m}$ & Muito crítico \\
\hline 6 & 0 lux & $50 \mathrm{~m}$ & Crítico \\
\hline 7 & $0 \operatorname{lux}$ & $7 \mathrm{~m}$ & Crítico \\
\hline 8 & $5 \operatorname{lux}$ & $20 \mathrm{~m}$ & Muito crítico \\
\hline 9 & $1 \operatorname{lux}$ & $20 \mathrm{~m}$ & Muito crítico \\
\hline 10 & 0 lux & $6 \mathrm{~m}$ & Crítico \\
\hline 11 & $0 \operatorname{lux}$ & 4 a $10 m$ & Crítico \\
\hline 12 & $0 \operatorname{lux}$ & $5,5 \mathrm{~m}$ & Crítico \\
\hline 13 & $2 \operatorname{lux}$ & $11 \mathrm{~m}$ & Muito crítico \\
\hline 14 & $0 \operatorname{lux}$ & $5 \mathrm{~m}$ & Crítico \\
\hline 15 & $0 \operatorname{lux}$ & $4 m$ & Crítico \\
\hline 16 & $1 \operatorname{lux}$ & $4 m$ & Muito crítico \\
\hline 17 & $1 \operatorname{lux}$ & $5 \mathrm{~m}$ & Muito crítico \\
\hline 18 & 6 lux & $18 \mathrm{~m}$ & Muito crítico \\
\hline 19 & $34 \operatorname{lux}$ & 0 & Muito crítico \\
\hline 20 & $34 \operatorname{lux}$ & $25 \mathrm{~m}$ & Muito crítico \\
\hline
\end{tabular}

\section{DISCUSSÃO}

Ocorreu desorientação e falta de orientação para a maioria dos filhotes de E. imbricata submetidos aos experimentos com iluminação artificial. Os dados desse trabalho corroboram com outros estudos feitos com outras espécies de tartarugas marinhas, comprovando que o processo natural de orientação dos filhotes é interrompido e/ou alterado na presença de iluminação artificial (MROSOVSKY et al., 1967; SALMON et al., 1995; KARNAD et al., 2009; RIVAS et al., 2015; PRICE et al., 2018). Os filhotes que foram considerados "desorientados" andaram em várias direções dentro da arena. Esse comportamento de andar em círculos ocorre quando as tartarugas são confrontadas com múltiplos estímulos que se equivalem (LIMPUS et al., 2013). No fim, estes filhotes podem ou não encontrar o caminho para o oceano, mas foram enquadrados em apenas uma categoria devido às consequências que ambos os casos podem trazer aos filhotes. De acordo com Lohmann et al. (1996) os filhotes que ficam andando em diversas direções e que não encontram o caminho para o oceano ficam expostos à ação de predadores como raposas e caranguejos. Já no caso dos que andam em várias direções, mas que encontram o caminho para o oceano, Wyneken (2000) explica que eles terminam gastando muita energia nesse percurso e, ao entrar na água, assumem um modo natatório mais lento que os que se orientam diretamente para o oceano, o que faz com que demorem mais a chegar à região de mar aberto, ficando assim, mais expostos a ação de predadores como peixes e outros organismos.

A literatura não evidencia a quantidade de filhotes como uma variável importante. Nos estudos já realizados os experimentos possuem número de filhotes bem variados, entre 2 e 60 indivíduos (SALMON et al., 1995; PENDOLEY et al., 2016). De acordo com Lohmann et al. (1997), os filhotes costumam emergir dos seus ninhos em grandes grupos, por tanto, para esse estudo, foram escolhidos os valores de n como 7 e 15 filhotes para verificar se a quantidade de filhotes influencia no processo de orientação. Foi encontrada diferença significativa entre os experimentos envolvendo $n=7$ filhotes e $n=15$ filhotes em relação às categorias de orientação. No escuro a maioria dos filhotes orientaram e no claro houve falta de orientação, o que podemos inferir que um dos fatores que poderia contribuir na orientação sem a poluição luminosa seria a quantidade de filhotes que emergem dos ninhos simultaneamente. De uma maneira geral, há necessidade de ajuste na metodologia para ter um protocolo quanto ao número de filhotes utilizados nos experimentos, bem como no tamanho das arenas, para ser possível uma análise comparativa entre as áreas 
de nidificação de tartarugas marinhas que sofrem com a iluminação artificial no mundo.

Após eclodirem dos ovos, os filhotes podem demorar de um até sete dias para emergirem dos ninhos (CHRISTENS, 1990). Isso ocorre porque esses animais têm mais atividade durante a noite, quando há uma diminuição na temperatura e, durante o dia, onde a temperatura é mais elevada costumam ficar imóveis para guardar energia (BUSTARD, 1967). Bustard (1967) explica que devido a isso, a maioria dos casos de emersão de filhotes dos ninhos é durante a noite. Nos experimentos realizados em Ipojuca, a temperatura, obteve correlação quanto ao número de filhotes envolvidos nos processos de orientação. Porém, não foi encontrado na literatura evidências que corroborassem com os dados ou que mostrassem que a temperatura influencia no processo natural de orientação dos filhotes. Não houve correlação quanto à velocidade do vento e umidade relativa do ar, possivelmente porque esses parâmetros não são tão variáveis durante a temporada.

Os resultados do presente estudo mostraram que há correlação entre a intensidade luminosa e a interrupção do processo natural de orientação dos filhotes. 0 mesmo resultado foi obtido em estudo realizado por Robertson et al. (2016) em Queensland/Austrália com filhotes de C. caretta. Os autores mostraram que, à medida que mais luzes eram acesas aumentando a intensidade luminosa, mais filhotes tinham sua orientação interrompida. Porém, alguns autores não atribuem esse fato apenas a intensidade da iluminação artificial (MROSOVSKY et al., 1968; SALMON et al., 1995). Salmon et al. (1995) sugerem que as diferenças de como as tartarugas respondem a iluminação artificial devem ser atribuídas a uma combinação entre a intensidade dessa iluminação e a sua composição. Vale salientar, que não há na literatura experimentos realizados com E. imbricata que especifique qual tipo de iluminação a espécie mais responde, necessitando de estudos que possam testar essa hipótese.

No presente estudo não foi obtido correlação entre as fases da lua e o processo de orientação em todos os casos testados. Porém, outros estudos mostram o contrário. Em Salmon et al. (1995) foi mostrado em seu trabalho com $C$. caretta, que nos experimentos realizados em áreas sob influência de iluminação artificial em noites onde a lua não estava visível, o processo natural de orientação dos filhotes foi interrompido, enquanto que, em noites com a presença da lua, esse processo era restaurado. Estes ainda observaram que, na medida em que a lua subia no horizonte e a intensidade de sua iluminação natural refletida no mar aumentava, o número de filhotes desorientados diminuía. Porém, estes afirmaram que, em algumas áreas sob forte influência da iluminação artificial, nem a presença da lua cheia era capaz de restaurar o processo de orientação dos filhotes. Resultados similares foram obtidos nos experimentos conduzidos por Robertson et al. (2016) também com a espécie $C$. caretta. Os autores mostraram que, quando a lua não estava presente e as luzes ligadas, apenas $21 \%$ dos filhotes orientaram corretamente. Na presença da lua cheia, essa proporção aumentou para $89 \%$. Esses resultados corroboram com muitos outros estudos (WITHERINGTON et al., 1991a; SALMON et al., 1995; ADAMANY et al., 1997; LOHMANN et al., 1997; TUXBURY et al., 2005; BOURGEOIS et al., 2009; BERRY et al., 2013).

É possível observar um alto número de filhotes entre as categorias de orientação na lua crescente, tanto para os experimentos realizados no claro como no escuro. Esse alto número pode ser explicado devido 
à realização de 6 experimentos para esta fase da lua para ambas situações, claro e escuro. Além disso, 24,24\% dos filhotes orientaram corretamente em experimentos realizados no claro com a lua cheia e crescente, evidenciando a influência lunar neste processo, como mostrado em outros estudos (SALMON et al., 1995; ROBERTSON et al., 2016). Porém, para a maioria dos filhotes $(75,76 \%)$ a luz da lua não foi o suficiente para restaurar sua orientação. Também é possível notar que o menor número de orientados e o maior número de desorientados e falta de orientação no escuro ocorreu na lua nova, o que mostra que a presença da lua também ajuda a guiar os filhotes mesmo em locais sem influência direta da iluminação artificial. A presença de filhotes desorientados e com falta de orientação nos experimentos realizados no escuro pode ser explicada devido à presença de iluminação artificial em locais distantes, mas visíveis no local onde foi realizado o experimento. O mesmo aconteceu em Robertson et al. (2016), onde a orientação dos filhotes foi influenciada pela presença da luz na cidade que ficava entre 2 e $4 \mathrm{~km}$ distante da praia onde foram realizados os experimentos.

A ausência de nidificação na praia de Porto de Galinhas, que tem a maior área de iluminação, pode ser atribuída a dois fatores: 1- Por ser uma área iluminada há décadas não é mais escolhida pelas tartarugas; 2- Pela presença dos recifes de corais existentes no local (MOURA et al., 2012), dificultando as tartarugas irem desovar na maré baixa. A praia de Merepe obteve a maior quantidade de pontos iluminados, pois nesse trecho é onde há a maior concentração de pousadas e hotéis além de ser a maior praia estudada, com extensão de 3,47km, enquanto que Muro Alto apresenta 2,50km de extensão, Cupe 2,37km e Maracaípe $3,11 \mathrm{~km}$ (MOURA et al., 2012).

Os pontos classificados como 'Muito crítico' obtiveram intensidade luminosa superior a 0 lux. Já os pontos 'Críticos' obtiveram sua intensidade luminosa igual a 0 lux, porém há registros anteriores feitos pela ONG Ecoassociados de casos de filhotes com o comportamento de desorientação e/ou falta de orientação, apresentados nesse estudo, no local. Isso pode ser explicado pelo fato que as luzes que obtiveram intensidade luminosa de 0 lux estavam a uma certa distância do local de nidificação, além de estarem voltadas para a praia e, como relatado, luzes mesmo distantes são capazes de interromper o processo de orientação dos filhotes (ROBERTSON et al., 2016).

Internacionalmente, com o intuito de mitigar os efeitos negativos causados pela iluminação artificial, algumas lâmpadas são vendidas ditas como 'turtle-friendly'. No estudo realizado por Robertson et al. (2016), duas lâmpadas do tipo "turtle-friendly" foram testadas. Uma foi a luz "LED Amber bulb", uma luz amarelada que foi feita para ser usada em casas. A outra luz foi a "red LED bulb", uma luz vermelha que é utilizada por pessoas que precisam andar na praia durante a noite, como nos monitoramentos noturnos, por exemplo. No geral, os experimentos mostraram que a luz amarela provocou o que foi considerado no presente estudo como "falta de orientação", principalmente quando havia mais de uma luz acesa no local. Para a luz vermelha, foi visto o comportamento aqui considerado com 'desorientação', com muitos filhotes andando em círculos.

As luzes testadas por Robertson et al. (2016), tanto a amarela como a vermelha, foram feitas para a costa leste dos Estados Unidos onde a orientação dos filhotes não sofre alteração com a utilização delas (WITHERINGTON et al., 1991a; WITHERINGTON et al., 1991b). As mesmas luzes testadas na costa da 
Austrália, com a mesma espécie de tartaruga marinha (C. caretta) afetou a orientação dos filhotes. Isso mostrou que, mesmo sendo da mesma espécie, populações diferentes podem responder de diferentes maneiras (ROBERTSON et al., 2016). Por tanto, os autores acima sugerem que decisões sobre a iluminação artificial devem ser feitas localmente, levando em consideração as características físicas da praia, o tipo de luz utilizada, a intensidade dessa iluminação e a resposta dos filhotes que nascem naquela região. Além disso, eles afirmam que o grande problema da iluminação artificial nas praias de nidificação de tartarugas marinhas é que o espectro visível das tartarugas marinhas se sobrepõe completamente ao espectro visível humano, por isso é impossível projetar uma luz que seja altamente visível para os seres humanos, mas invisível para as tartarugas marinhas. Por tanto, sempre que possível, deve-se evitar qualquer tipo de iluminação nas praias onde ocorrem desovas de tartarugas marinhas, principalmente na época de nidificação.

\section{CONCLUSÕES}

Os resultados do presente estudo mostraram que a luz artificial na praia do Ipojuca vem causando fortes impactos no processo de orientação dos filhotes de tartarugas marinhas e que essa influência negativa pode ser alta ao ponto de inibir os efeitos da iluminação natural da lua para a maioria dos filhotes. Entre os parâmetros abióticos analisados (luz, fases da lua, temperatura, umidade do ar e velocidade do vento) a intensidade da luz artificial foi a que expressou uma maior relação quanto as categorias analisadas. Ao longo dos $12 \mathrm{~km}$ da área de estudo, foram contabilizados 20 pontos sob a influência de iluminação artificial. Isso equivale a uma média de um ponto a cada $600 \mathrm{~m}$. Isso se torna um fato alarmante visto que para essa área há uma média de 150 ninhos de E. imbricata em cada temporada reprodutiva sem contar com os ninhos das outras espécies que também desovam na região, de acordo com a ONG Ecoassociados.

A fim de mitigar os efeitos causados pela foto poluição local, recomendamos que os proprietários de empreendimentos a beira mar sejam estimulados a apagarem as luzes durante a noite, principalmente na época de nidificação. Uma outra sugestão, caso haja a necessidade de manter a iluminação, é que essa possa ser adequada, mas para isso mais testes precisam ser realizados para descobrir qual é o tipo de luz que as espécies que nidificam na região respondem e encontrar um tipo que cause o menor impacto possível. Além do mais, deve existir apoio da gestão pública local com fiscalizações em relação a iluminação dos empreendimentos, bem como uma estreita parceria com os trabalhos da ONG Ecoassociados que realiza o monitorando das praias da região a mais de 20 anos.

\section{REFERÊNCIAS}

ADAMANY, S. L.; SALMON, M.; WITHERINGTON, B. E.. Behavior of sea turtles at an urban beach. III. Costs and benefits of nest caging as a management strategy. Florida Scientist, v.60, n.4, p.239-253, 1997.

BERRY, M.; BOOTH, D. T.; LIMPUS, C. J.. Artificial lighting and disrupted sea-finding behaviour in hatchling loggerhead turtles (Caretta caretta) on the Woongarra coast, south-east Queensland, Australia. Australian Journal of Zoology, v.61, p.137-145, 2013. DOI: https://doi.org/10.1071/ZO13028
BOURGEOIS, S.; GILOT-FROMONT, E.; VIALLEFONT, A.; BOUSSAMBA, F.; DEEM, S. L.. Influence of artificial lights, logs and erosion on Leatherback Sea Turtle hatchling orientation at Pongara National Park, Gabon. Biological Conservation, v.142, p.85-93, 2009. DOI: https://doi.org/10.1016/j.biocon.2008.09.028

BRASIL. Portaria n. 11, de 30 de janeiro de 1995. Proíbe qualquer fonte de iluminação com intensidade luminosa superior a zero lux em áreas de desovas de tartarugas marinhas. Brasília: DOU, 1995. 
BUSTARD, H. R.. Mechanism of nocturnal emergence from the nest in Green Turtle hatchlings. Nature, v.214, p.317317, 1967. DOI: https://doi.org/10.1038/214317a0

CHRISTENS, E.. Nest emergence lag in Loggerhead Sea Turtles. Journal of Herpetology, v.24, p.400-402, 1990. DOI: https://doi.org/10.2307/1565057

CRUZ, L. M.; SHILLINGER, G. L.; ROBINSON, N. J.; TOMILLO, P. S.; PALADINO, F. V.. Effect of light intensity and wavelength on the in-water orientation of Olive Ridley Turtle hatchlings. Journal of Experimental Marine Biology and

Ecology, v.505, p.52-56, 2018. DOI: https://doi.org/10.1016/j.jembe.2018.05.002

GUIMARÃES, E. S.; MOURA, G. J. B.; LINS, E. A. M.. Aspectos ecológicos de Eretmochelys imbricata entre os anos $2000 \mathrm{e}$ 2008 nas praias de Ipojuca-PE e lista comentada das espécies de tartarugas marinhas ocorrentes no Estado de Pernambuco. In: MOURA, G. J. B.; SANTOS, E. M.; OLIVEIRA, M. A. B.; CABRAL, M. C. C.. Herpetologia do Estado de Pernambuco. Brasília: Ministério do Meio Ambiente, 2010. p.305-317.

IUCN. International Union for the Conservation of Nature. Red List of Threatened Species, version 2019-3. IUCN, 2019.

KARNAD, D.; ISVARAN, K.; KAR, C. S.; SHANKER, K.. Lighting the way: Towards reducing misorientation of Olive Riddle hatchlings due to artificial lighting at Rushikulya, India. Biological Conservation, v.142, p.2083-2088, 2009. DOI: https://doi.org/10.1016/j.biocon.2009.04.004

LIMA, P. C. S.. Desenvolvimento local e turismo do Polo de Porto de Galinhas. Dissertação (Mestrado em Arquitetura e Urbanismo) - Universidade de Brasília, Brasília, 2006.

LIMPUS, C. J.; KAMROWSKI, R. L.. Ocean-finding in marine turtles: the importance of low horizon elevation as an orientation cue. Behaviour, v.150, p.863-893, 2013. DOI: https://doi.org/10.1163/1568539X-00003083

LOHMANN, K. J.; WITHERINGTON, B. E.; LOHMANN, C. M.; SALMON, M.. Orientation, navigation and natal beach homing in sea turtles. In: LUTZ, P. L.; MUSICK, J. A.. The biology of sea turtles. Tallahassee: CRC Press, 1997. p.107136.

LOHMANN, K.; LOHMANN, C.. Orientation and open-sea navigation in sea turtles. Journal of Experimental Biology, v.199, p.73-81, 1996.

MARCOVALDI, M. Â.; LOPEZ, G. G.; SOARES, L.; BELINI, C.; DOS SANTOS, A. S.; LOPEZ, M.. Avaliação da tartaruga marinha Eretmochelys imbricata (Linnaeus, 1766) no Brasil. Biodiversidade Brasileira, v.1, p.20, 2011.

MCFARLANE, R. W.. Disorientation of Loggerhead Hatchlings by artificial road lighting. Copiea, v.1963, p.153, 1963.

MMA. Ministério do Meio Ambiente. Livro Vermelho da Fauna Brasileira Ameaçada de Extinção: Volume IV Répteis. Brasília: MMA, 2018.

MORTIMER, J. A.; DONELLY, M.. Eretmochelys imbricata. The IUCN Red List of Threatened Species. 2008.
MOURA, C. C. D. M.; GUIMARÃES, E. D. S.; MOURA, G. J. B.; AMARAL, G. A.; SILVA, A. C.. Distribuição espaço-temporal e sucesso reprodutivo de Eretmochelys imbricata nas praias do Ipojuca, Pernambuco, Brasil. Ilheringia, Série Zoologia, v.102, p.254-260, 2012. DOI: https://doi.org/10.1590/S0073$\underline{47212012005000003}$

MROSOVSKY, N.; CARR, A.. Preference for light of short wavelengths in hatchling Green Sea Turtles, Chelonia mydas, tested on their natural nesting beaches. Behaviour, v.28, p.217-231, 1967. DOI: https://doi.org/10.1163/156853967X00019

MROSOVSKY, N.; SHETTLEWORTH, S. J.. Wavelength preferences and brightness cues in the water finding behaviour of sea turtles. Behaviour, v.32, p.211-257, 1968. DOI: https://doi.org/10.1163/156853968X00216

MROSOVSKY, N.; KINGSMILL, F.. How turtle find the sea. Ethology, v.67, p.237-256, 1985. DOI: https://doi.org/10.1111/i.1439-0310.1985.tb01392.x

PENDOLEY, K.; KAMROWSKI, R. L.. Influence of horizon elevation on the sea-finding behaviour of hatchling flatback turtles exposed to artificial light glow. Marine Ecology Progress Series, v.529, p.279-288, 2015. DOI: https://doi.org/10.3354/meps11305

PENDOLEY, K.; KAMROWSKI, R. L.. Sea-finding in marine turtle hatchlings: What is an appropriate exclusion zone to limit disruptive impacts of industrial light at night?. Journal for Nature Conservation, v.30, p.1-11, 2016. DOI: https://doi.org/10.1016/j.jnc.2015.12.005

PRICE, J. T.; BRUCE, D.; DOMANGUE, R. J.; PALADINO, F. V.. Exploring the role of artificial lighting in loggerhead turtle (Caretta caretta) nest-site selection and hatchling disorientation. Herpetological Conservation and Biology, v.13, p.415-422, 2018.

RIVAS, M. L.; TOMILLO, P. S.; URIBEONDO, J. D.; MARCO, A. Leatherback hatchling sea-finding in response to artificial lighting: Interaction between wavelength and moonlight. Journal of Experimental Marine Biology and Ecology, v.463, p.143-149, 2015. DOI: https://doi.org/10.1016/j.jembe.2014.12.001

ROBERTSON, K.; BOOTH, D. T.; LIMPUS, C. J.. An assessment of 'turtle-friendly'lights on the sea-finding behaviour of Loggerhead Turtle hatchlings (Caretta caretta). Wildlife Research, v.43, n.1, p.27-37, 2016. DOI: https://doi.org/10.1071/WR15138

SALIÉS, E.; LARA, P. H.; PAZETTO, F.; VERÍSSIMO, L. F.; ABREU, J. A.; SOARES, L. S.. Cartilha de fotopoluição. Fundação Pró TAMAR. 2014.

SALMON, M.; WITHERINGTON, B. E.. Artificial Lighting and Sea finding by Loggerhead Hatchlings: Evidence for Lunar Modulation. Copeia, v.1995, p.931-938, 1995. DOI: $10.2307 / 1447042$

SILVA, K. O.; SANTOS, E. M.; SIMÕES, T. N.; SILVA, A. C.. Encalhes de tartarugas marinhas no litoral sul de Pernambuco, Brasil. Revista Ibero Americana de Ciências ambientais, v.10, n.2, p.53-64, 2019. 
DOI: https://doi.org/10.6008/CBPC2179$\underline{6858.2019 .002 .0006}$

SIMÕES, T. N.; SILVA, A. C.; MOURA, C. C. M.. Influence of artificial lights on the orientation of hatchlings of

Eretmochelys imbricata in Pernambuco, Brazil. Zoologia, Curitiba, v.34, p.e13727, 2017. DOI:

https://doi.org/10.3897/zoologia.34.e13727

SIMÕES, T. N.; SILVA, A. C.; SANTOS, E. M.; CHAGAS, C. A.. Temperatura de incubação e razão sexual em filhotes recémeclodidos da tartaruga marinha Eretmochelys imbricata (Linnaeus, 1766) no município do Ipojuca, Pernambuco, Brasil. Papéis Avulsos de Zoologia, v.54, p.363-374, 2014. DOI: https://doi.org/10.1590/0031-1049.2014.54.25

TUXBURY, S. M.; SALMON, M.. Competitive interactions between artificial lighting and natural cues during seafinding by hatchling marine turtles. Biological Conservation, v.121, p.311-316, 2005. DOI:

http://doi.org/10.1016/j.biocon.2004.04.022
WITHERINGTON, B. E.; BJORNDAL, K. A.. Influences of artificial lighting on the seaward orientation of hatchling Loggerhead Turtles Caretta caretta. Biological Conservation, v.55, p.139-149, 1991a. DOI: https://doi.org/10.1016/00063207(91)90053-C

WITHERINGTON, B. E.; BJORNDAL, K. A.. Influences of wavelength and intensity on hatchling sea turtle phototaxis: implications for sea-finding behavior. Copeia, v.1991, p.1060-1069, 1991b. DOI: http://doi.org/10.2307/1446101

WYNEKEN, J.. The migratory behaviour of hatchling sea turtles beyond the beach. In: PILCHER, N.; ISMAIL, G.. Sea Turtles of the Indo-Pacific. ASEAN Academic Press, 2000. p.121-129.

ZHELEVA, M.. The dark side of light. Light pollution kills Leatherback Turtle hatchlings. BioDiscovery, v.3, p.e8930, 2012. DOI: http://doi.org/10.7750/BioDiscovery.2012.3.4

A CBPC - Companhia Brasileira de Produção Científica (CNPJ: 11.221.422/0001-03) detém os direitos materiais desta publicação. Os direitos referem-se à publicação do trabalho em qualquer parte do mundo, incluindo os direitos às renovações, expansões e disseminações da contribuição, bem como outros direitos subsidiários. Todos os trabalhos publicados eletronicamente poderão posteriormente ser publicados em coletâneas impressas sob coordenação da Sustenere Publishing, da Companhia Brasileira de Produção Científica e seus parceiros autorizados. Os (as) autores (as) preservam os direitos autorais, mas não têm permissão para a publicação da contribuição em outro meio, impresso ou digital, em português ou em tradução. 